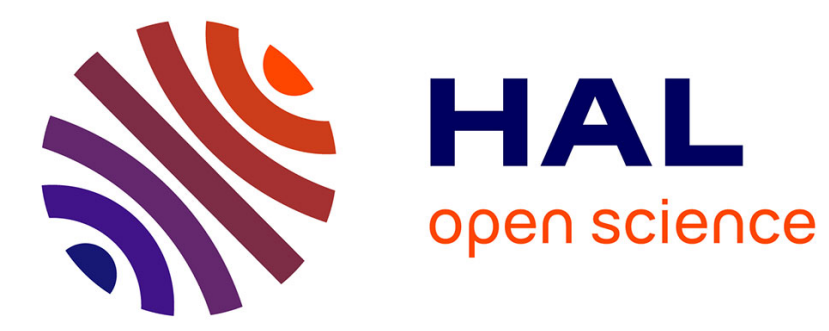

\title{
A Method for Flexible Aircraft LFT Modelling
}

\author{
J. Torralba, F. Demourant, G. Puyrou, G. Ferreres
}

\section{To cite this version:}

J. Torralba, F. Demourant, G. Puyrou, G. Ferreres. A Method for Flexible Aircraft LFT Modelling. European Control Conference, Aug 2009, BUDAPEST, Hungary. hal-01060318

\section{HAL Id: hal-01060318 \\ https://hal-onera.archives-ouvertes.fr/hal-01060318}

Submitted on 3 Sep 2014

HAL is a multi-disciplinary open access archive for the deposit and dissemination of scientific research documents, whether they are published or not. The documents may come from teaching and research institutions in France or abroad, or from public or private research centers.
L'archive ouverte pluridisciplinaire HAL, est destinée au dépôt et à la diffusion de documents scientifiques de niveau recherche, publiés ou non, émanant des établissements d'enseignement et de recherche français ou étrangers, des laboratoires publics ou privés. 


\title{
A Method for Flexible Aircraft LFT Modelling
}

\author{
J. Torralba and F. Demourant and G. Puyou and G. Ferreres
}

\begin{abstract}
This paper presents a method to obtain a Linear Fractional Transformation (LFT) model from a set of numerical flexible aircraft models. The core of the method lies in a polynomial interpolation, but before this step, some cautious treatments must be applied to the numerical models for them to be interpolable. First the models have to be reduced to be exploitable, while ensuring they all keep the same consistent modal content and avoiding numerical problems. Second the interpolation of the reduced models state representations can only be processed if the state vector has the same physical meaning for the whole model set (state vector consistency). For this problem, a specific state coordinate transformation is emphasized. After models are interpolated, the LFT is computed with the LFR toolbox's generalized Morton method. Then the LFT accuracy can be improved through a biconvex optimization.
\end{abstract}

\section{INTRODUCTION}

The Linear Fractional Transformation (LFT, see Fig. 1) modelling of aeroelastic (i.e. flexible) models was investigated in [1], [2] and [3] for flutter analysis. These methods are based upon the knowledge of the parametric structure of the aeroelastic equation of motion (1) to build the corresponding LFT with respect to flight parameters (e.g. Mach number) and mass cases. In this equation, $M, C$ and $K$ are respectively the mass, damping and stiffness of the aircraft structure. The aerodynamic contribution involves the dynamic pressure $q$ and the unsteady aerodynamic forces modelled by $Q$, which depends on flight parameters (Mach number and airspeed).

$$
M \ddot{\eta}+C \dot{\eta}+K \eta=q Q \eta
$$

In practice, this equation is evaluated at one flight point and one mass case and then the corresponding aeroelastic model is reduced. If a set of models is considered, generally the remaining modal content is not consistent from one flight point and mass case to another, leading to models with various number of states, and for which the parametric dependencies information has been lost. Hence the method that is presented in this paper, which is fully numeric as it is assumed that the only input data is a set of models $\left(G_{i}(s)\right)_{i \in[1, N]}$ describing the aeroelastic dynamics of the aircraft for a set of flight parameters and mass cases. In other words, the parametric structure is unknown a priori. Of course this method can also be used for other types of dynamic models for which the analytic structure is unknown.

It is worth pointing out that LFT models are deeply interesting for full flight domain aircraft control design (possibly

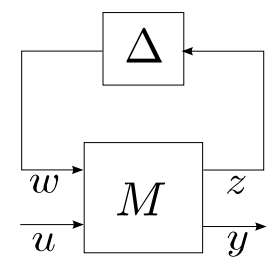

Fig. 1. LFT model structure

including flexible aspects). Indeed their intrinsic accounting for model parametric variations enables to directly design parameterized control laws (i.e. self-scheduling laws), which removes the industrially-famous tricky step of a posteriori interpolation of the laws. This way, LFT models may contribute towards simplifying present-day control design algorithms.

The paper is outlined as follows. Section II presents the overall algorithm: first a reduction method for a set of aeroelastic models is introduced, for the reduced models to be mutually consistent. An interesting state-space basis is also emphasized for the reduced models to be interpolable. Then an algorithm is proposed to optimize the LFT accuracy. Finally in section III, the algorithm is illustrated with a flexible aircraft LFT modelling example.

\section{ALGORITHM FOR FLEXIBLE AIRCRAFT LFT MODELLING}

Let us consider a set of linear models of a flexible aircraft $\left(G_{i}(s)\right)_{i \in[1, N]}$ corresponding to a set of values $\left(\delta^{i}\right)_{i \in[1, N]}$ of a vector $\delta$ gathering some significant flight and mass cases parameters (e.g. airspeed, Mach number, external fuel tank filling rate, ...).

We would like to compute an LFT model $F_{u}(M(s), \Delta)$ that matches this set of models (from both modal and frequential points of view), and that has sensible variations between the $N$ points. Hence the problem is to minimize the error $\max _{i \in[1, N]}\left\|F_{u}\left(M(s), \Delta^{i}\right)-G_{i}(s)\right\|_{\infty}$ with respect to $M(s)$ and to the model perturbation $\Delta=$ $\operatorname{diag}\left(\delta_{1} I_{i_{1}}, \ldots, \delta_{N} I_{i_{N}}\right)$ structure.

The guideline of the algorithm is to get a set of state representations $\left(A_{i}, B_{i}, C_{i}, D_{i}\right)_{i \in[1, N]}$ of the models $\left(G_{i}(s)\right)_{i \in[1, N]}$ that can be interpolated. This means that the state space matrices coefficients must be of same nature from one flight point to another. The algorithm then has roughly three steps : reduce the models number of states, make the models state representations interpolable, and interpolate them with respect to vector $\delta$ parameters, right before the LFT realization. These steps are explained hereafter. 


\section{A. Model reduction}

The reduction step is necessary to get exploitable models as flexible aircraft models generally have a lot of states, say 300 states. This reduction step is already a difficult task since the reduced models must be interpolable. So the reduction is required to provide models with the same number of modes, that must also be of same nature. There is no real formalism to declare that two modes for two different flight points are of same nature, this mainly relies on the physical sense of the engineer. But it can be said that a same mode varies with respect to the flight point and the mass case inside a restricted area of the complex plane (see Fig. 2(a)).

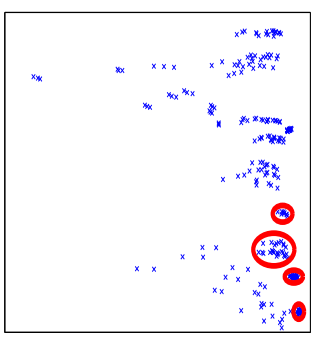

(a) Modes of same nature

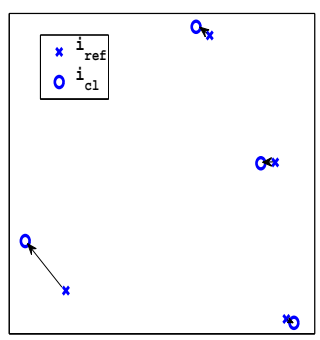

(b) Search of closest modes
Fig. 2. Consistent modes selection strategy

The well-known and efficient balanced reduction appears to difficult to use in this framework, because for each model the modes are modified according to observability and controlability properties, which destroys the overall modal consistency for the whole set of models.

Modal truncation is interesting because it avoids the problem of modal content alteration, but it cannot be directly used due to numerical problems linked with the complexity of the models. Finally, the best solution is to handle directly the modes of the models; the problem is hence to select a limited number of interesting modes, while ensuring that the $N$ sets of modes gather the same number of modes that are of same nature.

The first step for modes selection is to chose a reference model, say the one in the middle of the parametric domain (i.e. model number $i_{\text {ref }}$ ). For this model, only the $n_{x}$ modes inside the chosen bandwidth $f_{\max }$ are kept (eigenvalues $\lambda$ such that $|\lambda| \leq 2 \pi f_{\max }$ ); then it comes to find the closest flight point $i_{c l}$ to the reference one $\left(i_{c l}=\operatorname{argmin}_{i \in[1, N]}\left(\left\|\delta^{i_{r e f}}-\delta^{i}\right\|_{2}\right)\right)$, for the modes to be as close as possible to their locations at flight point $i_{\text {ref }}$ (the closest mode for model number $i_{c l}$ to a particular mode $\lambda_{k_{r e f}}^{i_{\text {ref }}}$ corresponding to $i_{\text {ref }}$ is $\lambda_{k_{c l}}^{i_{c l}}$ such that $k_{c l}=$ $\left.\operatorname{argmin}_{k \in\left[1, n_{x_{c l}}\right]}\left(\left|\lambda_{k_{r e f}}^{i_{\text {ref }}}-\lambda_{k}^{i_{c l}}\right|\right)\right)$. So for $i_{c l}$, the $n_{x}$ chosen modes are the closest ones to those corresponding to $i_{r e f}$ (see Fig. 2(b)). After flight point $i_{c l}$ is treated, the latter becomes the reference one and so on until the $N$ models are handled.
At this stage, having computed the $\mathrm{N}$ sets of modes, the $\mathrm{N}$ matrices $\left(A_{i}\right)_{i \in[1, N]}$ can be determined using a modal state representation :

$$
A_{i}^{\text {mod }}=\left(\begin{array}{cccc}
\Re\left(\lambda_{1}^{i}\right) & \Im\left(\lambda_{1}^{i}\right) & 0 & 0 \\
-\Im\left(\lambda_{1}^{i}\right) & \Re\left(\lambda_{1}^{i}\right) & 0 & 0 \\
0 & \ddots & \ddots & 0 \\
0 & 0 & \Re\left(\lambda_{n_{x}}^{i}\right) & \Im\left(\lambda_{n_{x}}^{i}\right) \\
0 & 0 & -\Im\left(\lambda_{n_{x}}^{i}\right) & \Re\left(\lambda_{n_{x}}^{i}\right)
\end{array}\right)
$$

Then it remains to find $\left(B_{i}, C_{i}, D_{i}\right)_{i \in[1, N]}$ matrices, by approximating the initial models $\left(G_{i}(s)\right)_{i \in[1, N]}$ frequency responses. This problem turns out to be a biconvex minimization of the Input/Output $(I / O)$ frequential error, which is noted $\Delta F_{i}(j \omega)=\left(C_{i}\left(j \omega I-A_{i}\right)^{-1} B_{i}+D_{i}-G_{i}(j \omega)\right)$. This quantity is used to define a frequential criterion that is expressed in two different manners: the first one (3) enables to minimize the error with respect to both $\left(B_{i}\right)_{i \in[1, N]}$ and $\left(D_{i}\right)_{i \in[1, N]}$, and the second one to $\left(C_{i}\right)_{i \in[1, N]}$ and $\left(D_{i}\right)_{i \in[1, N]}(4)$.

$$
\begin{aligned}
& J_{\Delta F^{*} \Delta F}=\cdots \\
& \sum_{i} \sum_{j} \operatorname{trace}\left(\Delta F_{i}^{*}\left(j \omega_{j}\right) \Delta F_{i}\left(j \omega_{j}\right)\right)\left(\omega_{j+1}-\omega_{j}\right)
\end{aligned}
$$

$$
\begin{aligned}
& J_{\Delta F \Delta F^{*}}=\cdots \\
& \sum_{i} \sum_{j} \operatorname{trace}\left(\Delta F_{i}\left(j \omega_{j}\right) \Delta F_{i}^{*}\left(j \omega_{j}\right)\right)\left(\omega_{j+1}-\omega_{j}\right)
\end{aligned}
$$

where $F^{*}$ denotes the conjugate transpose of matrix $F$.

The frequential error minimization algorithm will thoroughly be presented in section II-E.

This reduction method is attractive for two reasons. Firstly the modes are directly handled, which is convenient to sort them considering the whole set of models; secondly the biconvex optimization efficiently refines the frequency responses of the reduced models with respect to the reference models.

\section{B. Coordinate transformation for state vector consistency}

At this stage, the $N$ models are reduced, they have the same number of modes which are of same nature.

Now before interpolating the state space matrices, it is necessary to find a state basis ensuring these matrices are consistent in terms of states whatever flight point index $i$.

More precisely, after interpolation of the state matrices is done, the result must be regular modal trajectories as well as frequential responses variations with respect to flight parameters vector $\delta$ (see Fig. 3). This is an efficient test for state vector consistency.

Our research showed that the characteristic polynomial of the models (5) is of deep interest as regards the modal trajectories regularity constraint. This phenomenon can be explained by the physical nature of this polynomial's coefficients (6) that are directly linked with the model frequency 


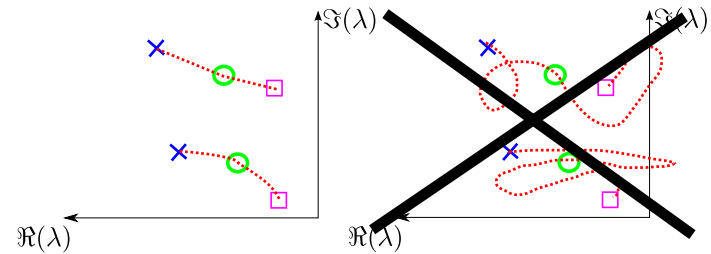

Fig. 3. A strong constraint : modal trajectories regularity

responses, and even more directly with the physical differential equations of the flexible aircraft.

$$
\begin{aligned}
& d(s)=\operatorname{det}(s I-A) \\
& d(s)=s^{n_{x}}+c_{n_{x}-1} s^{n_{x}-1}+\cdots+c_{1} s+c_{0}
\end{aligned}
$$

The state basis linked with the characteristic polynomial is the companion basis, in which the $A$ matrix has the form

$$
A_{i}^{c o m p}=\left(\begin{array}{ccccc}
0 & 1 & 0 & \cdots & 0 \\
0 & 0 & \ddots & \ddots & \vdots \\
\vdots & \vdots & \ldots & 1 & 0 \\
0 & 0 & \ldots & 0 & 1 \\
-c_{0}^{i} & -c_{1}^{i} & \ldots & \cdots & -c_{n_{x}-1}^{i}
\end{array}\right)
$$

This companion state basis is known to provide badlyconditioned state matrices. So it is necessary to regularize $A_{i}^{\text {comp }}$ matrices through a scaling matrix $T$ to balance the coefficients values, while keeping the same eigenvalues of course [4]. The same scaling is applied to all models (i.e. $\forall i$ ) for consistency. A regularized companion matrix $A_{i}^{\text {compr }}$ is then obtained (8).

$$
A_{i}^{\text {compr }}=T^{-1} A_{i}^{c o m p} T
$$

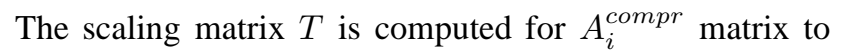
have the same rows and columns norms, as much as possible. More precisely, $T$ is a diagonal matrix gathering integer powers of two on its diagonal, to avoid roundoff errors (9).

$$
T=\operatorname{diag}\left(2^{k_{1}}, \ldots, 2^{k_{n_{x}}}\right)
$$

in which $\left(k_{p}\right)_{p \in\left[1, n_{x}\right]}$ are the aforementioned integers, independent of $i$ index (flight point index).

The final regularized companion matrix $A_{i}^{c o m p r}$ is then :

$$
A_{i}^{c o m p r}=\left(\begin{array}{cccc}
0 & 2^{k_{2}-k_{1}} & 0 & 0 \\
0 & 0 & \ddots & \vdots \\
0 & 0 & \cdots & 2^{k_{n_{x}}-k_{n_{x}-1}} \\
-c r_{0}^{i} & -c r_{1}^{i} & \cdots & -c r_{n_{x}-1}^{i}
\end{array}\right)
$$

where $\mathrm{cr}$ are the regularized characteristic polynomial coefficients.

Now the state space matrices corresponding to this regularized companion form (10) must be computed, knowing them in the modal basis (2).
The frequency response with respect to modal state matrices can be written :

$$
G_{i}(s)=C_{i}^{\text {mod }}\left(s I-A_{i}^{\text {mod }}\right)^{-1} B_{i}^{\text {mod }}+D_{i},
$$

and with respect to regularized companion state matrices :

$$
G_{i}(s)=C_{i}^{c o m p r}\left(s I-A_{i}^{c o m p r}\right)^{-1} B_{i}^{c o m p r}+D_{i} .
$$

The matrix $A^{\text {compr }}$ can readily be expressed in the modal basis (13), in three steps : first $A^{\text {compr }}$ is diagonalized, then the modes of the obtained $A$ matrix are reordered with respect to $A^{\text {mod }}$, and finally complex modes are split into real and imaginary parts, like in (2).

$$
A_{i}^{\text {compr }}=P_{i} A_{i}^{\text {mod }} P_{i}^{-1}
$$

From (12) and (13) we have :

$$
G_{i}(s)=C_{i}^{c o m p r} P_{i}\left(s I-A_{i}^{\text {mod }}\right)^{-1} P_{i}^{-1} B_{i}^{c o m p r}+D_{i} .
$$

And finally the companion state matrices $B_{i}^{\text {compr }}$ and $C_{i}^{c o m p r}$ are deduced from (11) and (14) $\left(A_{i}^{c o m p r}\right.$ and $D_{i}$ are already known) :

$$
\begin{aligned}
B_{i}^{\text {compr }} & =P_{i} B_{i}^{\text {mod }} \\
C_{i}^{\text {compr }} & =C_{i}^{\text {mod }} P_{i}^{-1}
\end{aligned}
$$

In this regularized companion basis, experiment shows that the state matrices $\left(A_{i}, B_{i}, C_{i}, D_{i}\right)_{i \in[1, N]}$ can be directly interpolated with respect to $\delta$ parameters, because of the modal trajectories regularity that is observed, meaning the state vector is consistent from one flight point to another.

\section{Models interpolation and LFT modelling}

1) Interpolation: The state space matrices are interpolated in their regularized companion form, through a multivariate polynomial structure. This problem can be easily solved with a least squares algorithm.

2) LFT realization: Once the interpolation structure is known, the LFT is simply realized with the generalized Morton's method [5] that is implanted in the LFR toolbox (function gmorton.m [6]). This method is the generalization of Morton's method to a polynomial expansion, and it lies on a singular values decomposition of each matricial coefficient.

\section{Validation of the LFT}

In order to assess the LFT accuracy, three criteria are defined : one evaluates the LFT modal matching with the reference models (16), and the other two are $H_{\infty}$ (17) and $\mathrm{H}_{2}$ (18) frequential criteria for the frequential matching assessment. 


$$
\epsilon_{\text {modal }}=\max _{i \in[1, N]}\left(\frac{\sum_{k=1}^{n_{x}}\left|\lambda_{k}^{i}-\lambda_{k}^{r e f, i}\right|}{\sum_{k=1}^{n_{x}}\left|\lambda_{k}^{\text {ref,i }}\right|}\right)
$$

where $\lambda_{k}^{i}$ is the LFT's k-th mode at flight point number $i$ and $\lambda_{k}^{r e f, i}$ refers to the corresponding reference model $G_{i}(s)$.

$$
\epsilon_{H_{\infty}}=\max _{i \in[1, N]}\left(\frac{\bar{\sigma}\left(\Delta F_{i}(j \omega)\right.}{\bar{\sigma}\left(G_{i}(j \omega)\right)}\right)
$$

in which $\Delta F_{i}(j \omega)=\left(F_{u}\left(M(j \omega), \Delta^{i}\right)-G_{i}(j \omega)\right)$ and $\bar{\sigma}$ is the maximum singular singular value on the pulsation continuum $\left(H_{\infty}\right.$ norm).

$$
\begin{aligned}
& \epsilon_{H_{2}}=\cdots \\
& \max _{i \in[1, N]}\left(\sqrt{\frac{1}{2 \pi} \frac{\sum_{j} \operatorname{trace}\left(\Delta F_{i}^{*}\left(j \omega_{j}\right) \Delta F_{i}\left(j \omega_{j}\right)\right) \Delta \omega_{j}}{\operatorname{trace}\left(G_{i}^{*}\left(j \omega_{j}\right) G_{i}\left(j \omega_{j}\right)\right)}}\right)
\end{aligned}
$$

where $\Delta \omega_{j}=\left(\omega_{j+1}-\omega_{j}\right)$.

In depth validation is of course necessary to check both modal and frequential behaviours of the LFT on the whole model continuum. This step will be illustrated in the applicative example.

\section{E. Input/Output error minimization}

If the $I / O$ error is not satisfactory, it can be minimized with a biconvex optimization. This algorithm is an extension to the LFT case of the one previously mentioned in paragraph II-A. In this situation, the minimized criterion (see (3) and (4)) depends on the frequential error between the LFT and the reference models :

$$
\Delta F_{i}(j \omega)=\left(F_{u}\left(M(j \omega), \Delta^{i}\right)-G_{i}(j \omega)\right)
$$

Let us recall the state representation of an LFT :

$$
\left\{\begin{array}{l}
\dot{x}=A x+B_{1} w+B_{2} u \\
z=C_{1} x+D_{11} w+D_{12} u \\
y=C_{2} x+D_{21} w+D_{22} u
\end{array}\right.
$$

The LFT frequency response is then (the model index $i$ is dropped for simplicity) :

$$
F_{u}(M(j \omega), \Delta)=\mathcal{C}(\Delta) Y(j \omega, \Delta) \mathcal{B}(\Delta)+\mathcal{D}(\Delta)
$$

with $Y(j \omega, \Delta)=(j \omega I-\mathcal{A}(\Delta))^{-1}, \mathcal{A}(\Delta)=A+B_{1} X_{\Delta} C_{1}$, $\mathcal{B}(\Delta)=B_{2}+B_{1} X_{\Delta} D_{12}, \mathcal{C}(\Delta)=C_{2}+D_{21} X_{\Delta} C_{1}, \mathcal{D}(\Delta)=$ $D_{22}+D_{21} X_{\Delta} D_{12}$, and $X_{\Delta}=\Delta\left(I-D_{11} \Delta\right)^{-1}$.

Hence the two expressions of the frequential error :

$$
\begin{aligned}
\Delta F(j \omega) & =\mathcal{H}_{B D}\left(\begin{array}{c}
B_{2} \\
D_{12} \\
D_{22}
\end{array}\right)-G(j \omega) \\
& =\left(\begin{array}{lll}
C_{2} & D_{21} & D_{22}
\end{array}\right) \mathcal{H}_{C D}-G(j \omega)
\end{aligned}
$$

with $\mathcal{H}_{B D}^{T}(j \omega)=\left(\begin{array}{c}Y^{T}(j \omega, \Delta) \mathcal{C}^{T}(\Delta) \\ X_{\Delta}^{T}\left[B_{1}^{T} Y^{T}(j \omega, \Delta) \mathcal{C}^{T}(\Delta)+D_{21}^{T}\right] \\ I\end{array}\right)$ and $\mathcal{H}_{C D}(j \omega)=\left(\begin{array}{c}Y(j \omega, \Delta) \mathcal{B}(\Delta) \\ X_{\Delta}\left[C_{1} Y(j \omega, \Delta) \mathcal{B}(\Delta)+D_{12}\right] \\ I\end{array}\right)$.

If the first set of variables of the biconvex optimization is written as :

$$
\left(\begin{array}{c}
B_{2} \\
D_{12} \\
D_{22}
\end{array}\right)=\left(\begin{array}{lll}
\beta_{1} & \ldots & \beta_{m}
\end{array}\right)
$$

where $\left(\beta_{k}\right)_{k \in[1, m]}$ are column vectors to be found, we shall then have :

$$
\begin{aligned}
& \operatorname{trace}\left(\Delta F^{*}(j \omega) \Delta F(j \omega)\right)=\cdots \\
& \sum_{k=1}^{m}\left(\beta_{k}^{T} Q \beta_{k}-2 \Re\left(l_{k}^{T}(j \omega)\right) \beta_{k}\right)+\operatorname{trace}\left(G^{*}(j \omega) G(j \omega)\right) \\
& \text { with } Q=\mathcal{H}_{B D}^{*}(j \omega) \mathcal{H}_{B D}(j \omega), G^{*}(j \omega) \mathcal{H}_{B D}(j \omega)= \\
& \left(\begin{array}{c}
l_{1}^{T}(j \omega) \\
\vdots \\
l_{m}^{T}(j \omega)
\end{array}\right) \text { and }\left(l_{k}\right)_{k \in[1, m]} \text { are column vectors. }
\end{aligned}
$$

Likewise, with the second set of variables as :

$$
\left(\begin{array}{ccc}
C_{2} & D_{21} & D_{22}
\end{array}\right)=\left(\begin{array}{c}
\gamma_{1}^{T} \\
\vdots \\
\gamma_{p}^{T}
\end{array}\right)
$$

we get :

$$
\begin{aligned}
& \operatorname{trace}\left(\Delta F(j \omega) \Delta F^{*}(j \omega)\right)=\cdots \\
& \sum_{k=1}^{p}\left(\gamma_{k}^{T} Q \gamma_{k}-2 \gamma_{k} \Re\left(h_{k}(j \omega)\right)\right)+\operatorname{trace}\left(G(j \omega) G^{*}(j \omega)\right) \\
& \text { with } Q=\mathcal{H}_{C D}(j \omega) \mathcal{H}_{C D}^{*}(j \omega), \mathcal{H}_{C D}(j \omega) G^{*}(j \omega)= \\
& \left(\begin{array}{c}
h_{1}^{T}(j \omega) \\
\cdots \\
h_{p}^{T}(j \omega)
\end{array}\right)^{T} \text { and }\left(h_{k}\right)_{k \in[1, p]} \text { are column vectors. }
\end{aligned}
$$

Back with models indices i.e. $i$, each term of both criterion to be minimized ((3) and (4)) has the following quadratic structure :

$$
\begin{aligned}
\operatorname{trace}\left(\Delta F_{i}\left(j \omega_{j}\right) \Delta F_{i}^{*}\left(j \omega_{j}\right)\right)= & \cdots \\
& c_{i, j}-2 \theta^{T} f_{i, j}+\theta^{T} Q_{i, j} \theta
\end{aligned}
$$

The final expression is a quadratic criterion $c-$ $2 \theta^{T} f+\theta^{T} Q \theta$, with $c=\sum_{i} \sum_{j} c_{i, j}\left(\omega_{j+1}-\omega_{j}\right), f=$ $\sum_{i} \sum_{j} f_{i, j}\left(\omega_{j+1}-\omega_{j}\right)$ and $Q=\sum_{i}^{i} \sum_{j} Q_{i, j}\left(\omega_{j+1}-\omega_{j}\right)$. There exists an analytical minimum at $\theta=Q^{\dagger} f$, which makes each loop of the biconvex optimization very fast.

\section{APPLICATION}

This application illustrates the previously presented method of LFT modelling from a set of numerical models corresponding to a set of flight point and mass cases. These models are aircraft longitudinal flexible models that are wanted to be modelled as an LFT for control design purpose. 


\section{A. Description of the model}

The set of aircraft models $\left(G_{i}(s)\right)_{i \in[1, N]}$ correspond to variations of the parameters $\delta=\left(\begin{array}{lll}O T & M a & V_{c}\end{array}\right)$, being respectively the outer tanks filling rate, Mach number and conventional airspeed.

These parameters vary inside the domain depicted in Fig. 4. For interpolation, $N=27$ points are chosen inside this parametric domain.

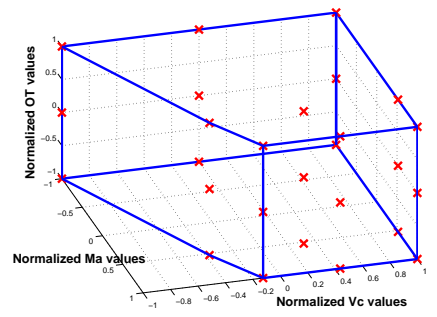

Fig. 4. LFT model representativity parametric domain. Solid lines: parametric domain; crosses: reference points for LFT modelling

The inputs of the model are the elevator $\delta q$ and the ailerons in symetric mode $\delta p_{\text {sym }}=\left(\delta p_{\text {left }}+\delta p_{\text {right }}\right)$ :

The outputs are critical wing loads i.e. bending load $W M X$ and twisting load $W M Y$ at wing root.

\section{B. Model reduction}

The intial state space flexible models have an average of 270 states. By setting the would-be LFT representativeness bandwidth to $8 \mathrm{~Hz}(\approx 50 \mathrm{rad} / \mathrm{s})$, the number of states drops to 18 i.e. 9 flexible modes (see Fig. 5).

When the 27 consistent sets of 9 modes are formed, it remains to reconstruct the $I / O$ responses of the initial models with this limited number of modes, to get the reduced models (an example of reduction result for one flight point is given in Fig. 5 and 6).

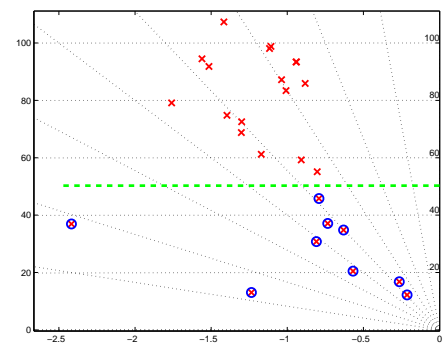

Fig. 5. Modal comparison of initial (x) and reduced models (o)

\section{LFT construction}

The LFT is then built according to the method presented in II-B, II-C and II-C.2. The polynomial terms used for interpolation are computed by expanding the polynomial $(1+\overline{O T})^{2}(1+\bar{M} a)^{2}\left(1+\bar{V}_{c}\right)^{2}$. The obtained LFT has the following $\Delta$-block :
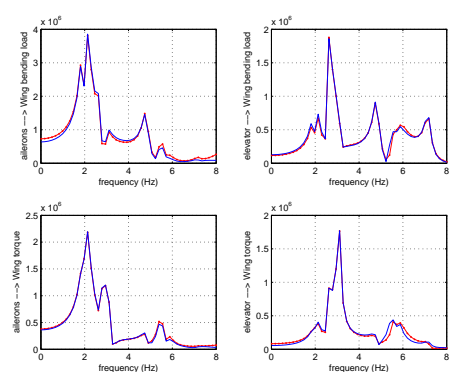

Fig. 6. Frequential comparison of initial (.-) and reduced models (-)

$$
\begin{aligned}
\Delta & =\operatorname{diag}\left(\overline{O T} \times I_{40}, \bar{M} a \times I_{30}, \bar{V}_{c} \times I_{40}\right) \\
\operatorname{dim}(\Delta) & =110
\end{aligned}
$$

\section{Validation of the LFT}

The values of the validation criteria (16), (17) and (18) for our example are gathered in table I.

TABLE I

LFT VALIDATION

\begin{tabular}{|c|c|c|}
\hline$\epsilon_{\text {modal }}(\%)$ & $\epsilon_{H_{\infty}}(\%)$ & $\epsilon_{H_{2}}(\%)$ \\
\hline $1.12 \times 10^{-8}$ & $3.83 \times 10^{-7}$ & $1.94 \times 10^{-7}$ \\
\hline
\end{tabular}

There is almost a perfect match between the LFT and the set of reference models $\left(G_{i}(s)\right)_{i \in[1,27]}$.

\section{E. Regularity check-up}

Since an LFT is a continuum of models, the previously built LFT has also to be checked-up between the flight points used to design it. It must be proven that the continuum of modes (i.e. modal trajectories when $\bar{\delta}$ varies) and the frequency response continuum are both regular. No "overshoot" must be observed, and ideally the continuum should vary linearly between two reference flight points.

In our application, the principal directions of the parametric domain are explored to assess the regularity properties of the LFT.

The modal trajectories (see Fig. 7) show that the LFT has no unexepected behaviour (i.e. no irregularities) in terms of modes. Besides, this proves the interest of characteristic polynomial coefficients for the state vector consistency in a set of models.

The frequency response continuum (Fig. 8) is fully satisfactory as well.

These tests are not thorough but the good properties of the LFT along the domain's principal directions, together with its matching the reference models, are good indicators of its validity on the whole continuum.

\section{F. Effect of error minimization algorithm}

Now the LFT has been validated in the wake of an exact interpolation, it can be interesting to attempt to decrease its complexity. There exists two ways to simplify the LFT 

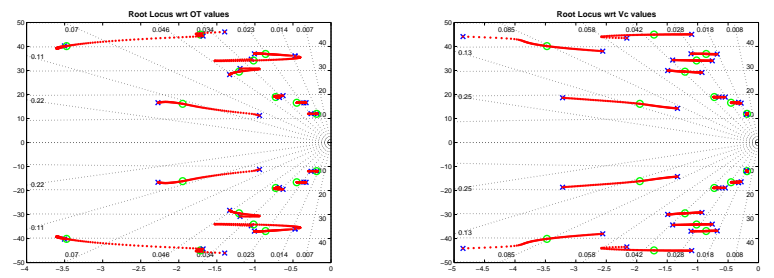

Fig. 7. Modal trajectories with respect to OT and $V_{c}$ parameters. x : reference models modes; o : central model modes
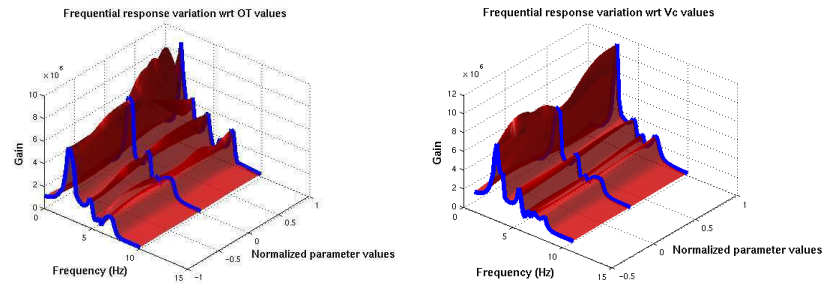

Fig. 8. frequency response continuum with respect to OT and $V_{c}$ parameters. Solid lines : reference models frequency responses

complexity : one consists in reducing the LFT a posteriori i.e. after the interpolation and the LFT realization (using gmorton.m), the second one is an a priori approach. It is the latter that will be detailed because it is more likely to reduce the LFT complexity, as the former (i.e. a posteriori reduction) is already included in gmorton.m (see LFR toolbox's function minlfr.m dealing with LFT complexity reduction inspired from balanced reduction).

So the solution focused here to get a lower-sized LFT is to simplify the interpolation formula, by sorting the polynomial terms and using only the most relevant ones through a correlation analysis between the latter variations and those of the coefficients to be interpolated.

The $\Delta$-block dimension decreases about linearly with respect to the number of polynomial terms used for interpolation (see Fig. 9); In the case of an exact interpolation, the error minimization algorithm (see II-E) is useless, but it becomes necessary in the approximation case. Indeed, as shown in Fig. 9, the algorithm dramatically reduces the frequential error; for example with 18 polynomial terms, the $\Delta$-block dimension reduction with respect to the exact interpolation case is $30 \%$, and the effect of the frequential error minimization is a $50 \%$ accuracy gain with respect to the rough interpolation result.

This LFT reduction algorithm has been used for multiobjective flight control law design in order to use both a relevant and a reasonably-sized LFT model for a robust design. Our LFT reduction algorithm based on polynomial terms sorting enabled a $50 \%$ drop of the design LFT size, and though a $30 \%$ loss of accuracy, final results showed the performance obtained with the analysis model was really close to those foreseen with this reduced design LFT model.

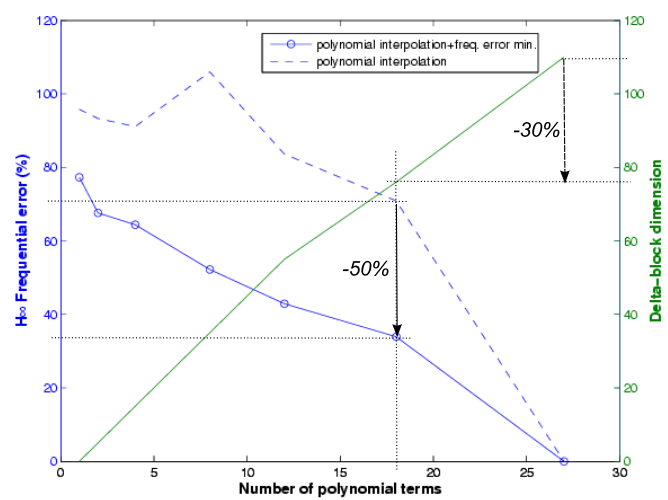

Fig. 9. Evolution of frequential error and $\Delta$-block dimension with respect to the polynomial interpolation complexity

\section{CONCLUSION}

The method presented in this paper proposes to design an LFT from a set of aeroelastic models. It is definitely adapted to complex and prominently numerical models, with no parametric structure knowledge whatsoever. The solution had then to involve as simple algorithms as possible to balance the data complexity. Naturally the least squares algorithm is used to interpolate the models with a basis of polynomials. Before interpolation, two steps are fateful in the process : the consistent reduction of the models and their state representations transformation in a regularized companion state basis. This way, the reduced models are made interpolable. After the LFT is realized through the generalized Morton's method, its $I / O$ accuracy can be optimized with an efficient biconvex optimization of the LFT state matrices. As mentioned earlier, LFT models are well-adapted to full flight domain aircraft control design. So these flexible LFT models are to be used in the framework of research on the promising multiobjective flexible aircraft control [7], [8], extended to the full flight domain case.

\section{REFERENCES}

[1] S. Bennani, B. Beuker, J. Willem van Staveren, and J.-P. Dijkgraaf, "Numeric LFT generation for robust aircraft flutter analysis," Proceedings of the IEEE CAC, September 2002.

[2] R. Lind, "Match-point solutions for robust flutter analysis," Journal of aircraft, vol. 39, pp. 91-99, 2002.

[3] C. Cumer, G. Ferreres, and M. Humbert, "Flutter detection with robustness tools," International Forum on Aeroelasticity and Structural Dynamics 2003, June 2003.

[4] B. Parlett and C. Reinsch, "Balancing a matrix for calculation of eigenvalues and eigenvectors," Numerische Mathematik, vol. 13, pp. 293-304, 1969.

[5] B. Morton, "New applications of $\mu$ to real-parameter variation problems," Proceedings of the 24th IEEE Conference on Decision and Control, pp. 233-238, December 1985.

[6] J. Magni, "User manual of the linear fractional representation toolbox, version 2. technical report TR 5/10403.01F DCSD, onera-cert: www.cert.fr/dcsd/idco/perso/magni/books.html," October 2005.

[7] S. Boyd and C. Barrat, Linear controller design: limits of performance. Prentice Hall, 1991.

[8] G. Ferreres and G. Puyou, "Flight control law design for a flexible aircraft: limits of performance," Journal of Guidance, Control, and Dynamics, vol. 29, pp. 870-878, July-August 2006. 